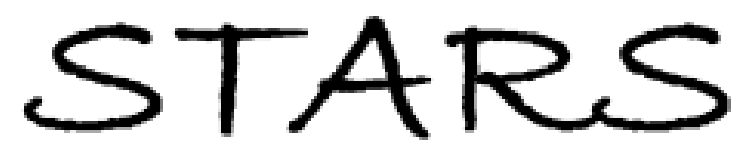

University of Central Florida

STARS

$1-1-2006$

\title{
Balancing detector effects with aberrations in the design of wide- field grazing incidence $x$-ray telescopes
}

James E. Harvey

University of Central Florida

Martina Atanassova

University of Central Florida

Andrey Krywonos

University of Central Florida

Find similar works at: https://stars.library.ucf.edu/facultybib2000

University of Central Florida Libraries http://library.ucf.edu

This Article; Proceedings Paper is brought to you for free and open access by the Faculty Bibliography at STARS. It has been accepted for inclusion in Faculty Bibliography 2000s by an authorized administrator of STARS. For more information, please contact STARS@ucf.edu.

\section{Recommended Citation}

Harvey, James E.; Atanassova, Martina; and Krywonos, Andrey, "Balancing detector effects with aberrations in the design of wide-field grazing incidence x-ray telescopes" (2006). Faculty Bibliography 2000s. 6212.

https://stars.library.ucf.edu/facultybib2000/6212

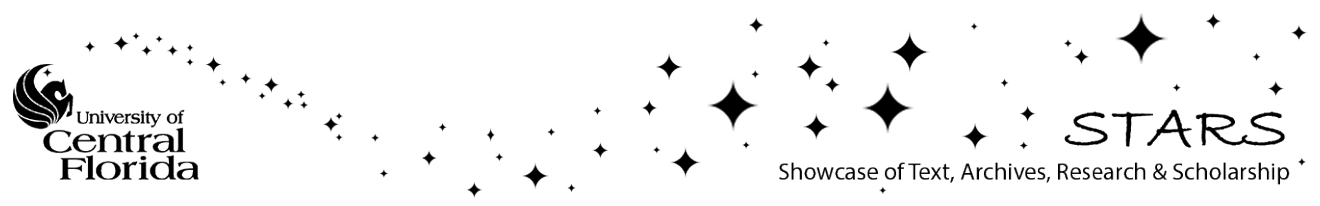




\section{Balancing detector effects with aberrations in the design of wide-field grazing incidence $x$-ray telescopes}

\author{
James E. Harvey \\ Martina Atanassova \\ Andrey Krywonos \\ University of Central Florida \\ Center for Research and Education in Optics \\ and Lasers (CREOL) \\ P.O. Box 162700 \\ 4000 Central Florida Boulevard \\ Orlando, Florida 32826
}

\begin{abstract}
Most imaging systems today include a mosaic detector array in the focal plane. Optical designers of astronomical telescopes typically produce a design that yields a superb on-axis aerial image in the focal plane, and detector effects are included only in the analysis of the final system performance. Aplanatic optical designs (corrected for spherical aberration and coma) are widely considered to be superior to nonaplanatic designs. However, there is little merit in an aplanatic design for wide-field applications because one needs to optimize some fieldweighted-average measure of resolution over the desired operational field of view (OFOV). Furthermore, when used with a mosaic detector array in the focal plane, detector effects eliminate the advantage of the aplanatic design even at small field angles. For wide fields of view, the focal plane is frequently despaced to balance field curvature with defocus thus obtaining better overall performance. We will demonstrate that including detector effects in the optical design process results in a different optimal (nonaplanatic) design for each OFOV that is even superior to an optimally despaced aplanatic design. () 2006 Society of Photo-Optical Instrumentation Engineers. [DOI: 10.1117/1.2209215]
\end{abstract}

Subject terms: detector effects; wide-field imaging systems; grazing incidence $\mathrm{x}$-ray telescopes.

Paper 050318R received Apr. 22, 2005; revised manuscript received Oct. 13 2005; accepted for publication Nov. 23, 2005; published online Jun. 9, 2006. This paper is a revision of a paper presented at the SPIE conference on Current Developments in Lens Design and Optical Engineering V, August 2004, Denver, Colorado. The paper presented there appears (unrefereed) in SPIE Proceedings Vol. 5523.

\section{Introduction and Historical Background}

The historical approach for designing astronomical (stellar) telescopes has been to first obtain a design corrected for spherical aberration, such as the classical Cassegrain design consisting of a paraboloidal primary mirror and a hyperboloidal secondary mirror. A perfect geometrical image of a star is thus produced if the telescope is precisely pointed such that the star lies on the optical axis. For the classical Cassegrain telescope, all off-axis images exhibit fielddependent aberrations such as coma, astigmatism, field curvature, and other higher-order aberrations. Since coma dominates astigmatism and field curvature for small field angles, the next step has historically been to correct coma, while maintaining the correction for spherical aberration. The telescope now produces a superb image on-axis and for a small field of view about the optical axis. This greatly relaxes the pointing tolerance for a stellar telescope and allows a cluster of stars, or a distant galaxy, to be imaged with high resolution. Optical systems corrected for both spherical aberration and coma are called aplanatic designs. The Ritchey-Chretien telescope consisting of a hyperboloidal primary mirror and a hyperboloidal secondary mirror is an aplanatic modification of the classical Cassegrain design. Historically, only after spherical aberration and coma

0091-3286/2006/\$22.00 @ 2006 SPIE are corrected would the designer of an astronomical telescope proceed to correct astigmatism (the anastigmat is an optical system corrected for spherical aberration, coma, and astigmatism) and field curvature, and a more complex three- or four-mirror system would be required to accomplish that goal. For the above reasons aplanatic optical designs are widely considered to be superior to nonaplanatic designs.

If a large field of view is desired from a two-mirror telescope, the focal plane of an aplanatic design is frequently despaced to balance small-field aberrations with large-field aberrations; i.e., field curvature is reduced at the large field angles at the expense of introducing defocus on-axis and for small field angles. Note that we make a distinction between the act of physically displacing or "despacing" an optical component or focal plane and the resulting "defocusing" effect it produces on the image.

Grazing incidence configurations are necessary to obtain sufficient reflectance for $\mathrm{x}$-ray telescopes. ${ }^{1}$ The classical Wolter type I design is somewhat analogous to the classical Cassegrain telescope as it consists of a grazing incidence paraboloidal primary mirror and a hyperboloidal secondary mirror and is inherently free of spherical aberration. ${ }^{2,3}$ The Wolter-Schwarzschild design is an aplanatic grazing incidence $\mathrm{x}$-ray telescope design consisting of two general aspheric surfaces that strictly satisfies the Abbe sine 


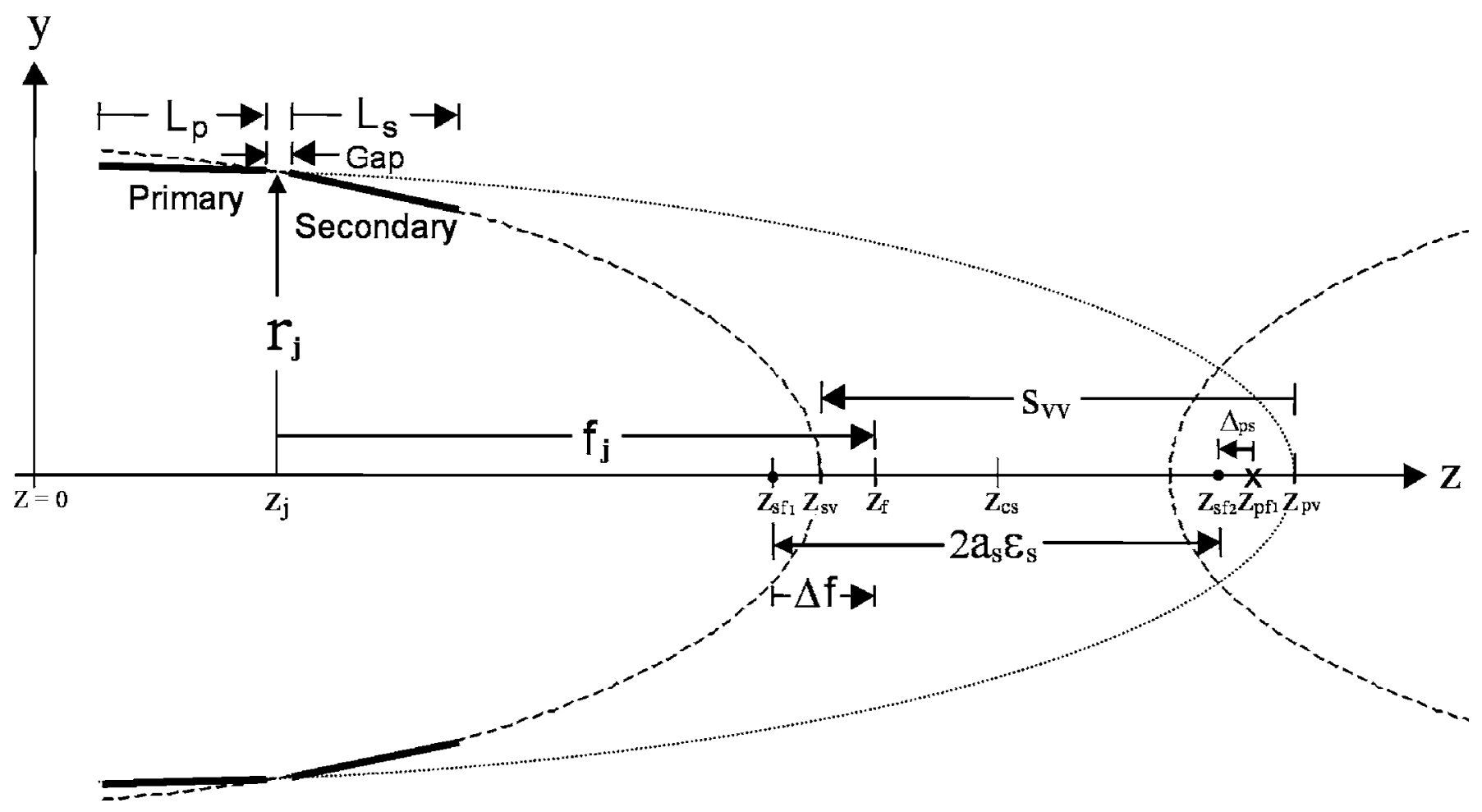

Fig. 1 Hyperboloid-hyperboloid grazing incidence $x$-ray telescope design.

condition. ${ }^{4,5}$ These grazing incidence telescopes suffer from much more severe aberrations than their conventional normal-incidence counterparts. ${ }^{6-9}$

For wide-field grazing incidence $\mathrm{x}$-ray telescopes, two considerations greatly diminish the advantage of an aplanatic optical design: (1) at these very small wavelengths $(6 \AA<\lambda<60 \AA)$, surface scatter effects dominate coma at small field angles, and (2) field curvature, astigmatism, and higher order aberrations dominate coma at large field angles. Hence there is little or no merit in using any of the precious few design variables in a two-mirror telescope to correct an aberration (coma) that has no dominant effect upon image quality. ${ }^{10}$ Furthermore, it has been shown that, if one chooses the field-weighted-average rms image size as a merit function, ${ }^{11}$ for large fields of view an optimum (nonaplanatic) hyperboloid-hyperboloid design outperforms the optimally despaced Wolter-Schwarzschild (aplanatic) optical design. ${ }^{12}$

A generalized Wolter type I grazing incidence x-ray telescope with a hyperboloid-hyperboloid optical prescription with the same first-order properties as the classical Wolter type I can be obtained by using the optimization capabilities of an optical design code such as ZEMAX or Code V. Five independent optical design parameters consisting of the vertex radii of curvature of the two mirrors, their conic constants, and the vertex-to-vertex separation $\left(R_{\mathrm{vp}}, \kappa_{\mathrm{p}}, R_{\mathrm{vs}}\right.$, $\kappa_{\mathrm{s}}$, and $S_{\mathrm{vv}}$ ) are required to completely characterize the hyperboloid-hyperboloid optical prescription shown in Fig. 1. In addition, the primary and secondary mirror lengths and the gap separating them must be specified $\left(L_{\mathrm{p}}, L_{\mathrm{s}}\right.$, and gap). The eccentricity $\varepsilon$, conic constant $\kappa$, and vertex radius of curvature $R_{\mathrm{v}}$ of a hyperboloid is related to the standard hyperboloid constants, $a=$ semimajor axis and $b$ =semiminor axis, by the following expressions

$\varepsilon=\sqrt{\frac{a^{2}}{b^{2}}+1}, \quad \kappa=-\varepsilon^{2}, \quad R_{v}=-b^{2} / a$.

The optimization merit function must contain constraints to preserve the system focal length $\left(f^{\prime}\right)$, the joint radius $\left(r_{\mathrm{j}}\right)$, and to assure that the joint grazing angle for the primary and secondary mirrors are the same. Note that the front focus of the primary mirror does not coincide with the rear focus of the secondary mirror as is the case with the classical Wolter type I design. This confocal delta is indicated as the quantity $\Delta_{\mathrm{ps}}$ in Fig. 1. Similarly, the system focal plane does not lie at the front focus of the secondary mirror. This displacement is indicated as $\Delta f$.

Reference 12 described in detail how an optimal parametric family of hyperboloid-hyperboloid grazing incidence X-ray telescope designs was developed with the firstorder properties of the Solar X-ray Imager (SXI). ${ }^{13}$ In the actual optimization process, we started with the classical Wolter type I design (paraboloid-hyperboloid) with the desired first-order parameters. We then chose three field angles; the center of the field $\left(\theta_{1}=0\right)$, an intermediate angle $\left(\theta_{B}\right)$, which will serve as the parameter that distinguishes between the various designs, and the edge of the desired field of view $\left(\theta_{3}=21\right.$ arc min). We then merely let the ZEMAX optimization routine work to minimize the rms spot size, letting it vary the available design parameters while maintaining the necessary system constraints. By changing the value of $\theta_{B}$ and repeating the process, we obtained the 


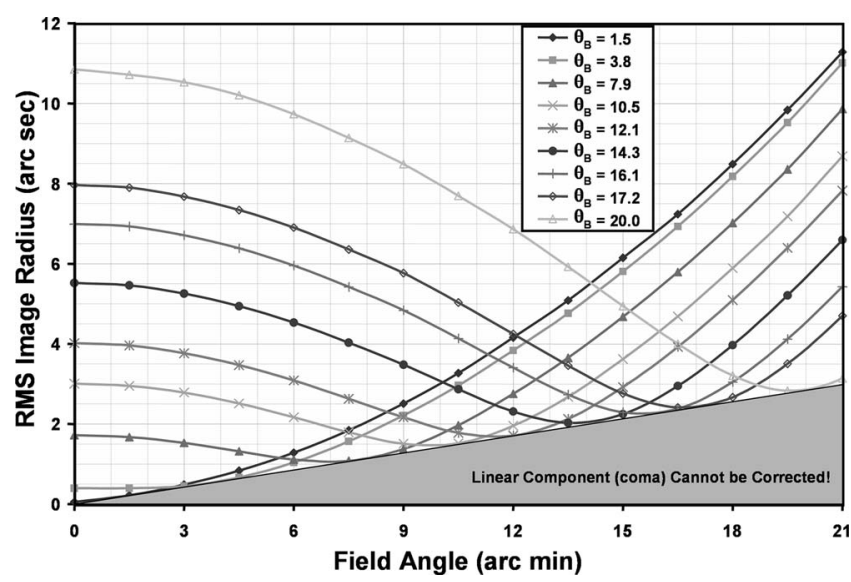

Fig. 2 Geometrical performance of optimal family of hyperboloidhyperboloid designs.

various members of the parametric family of designs. In each case we placed somewhat more weight on the intermediate angle $\theta_{B}$ than on the center and the edge of the field during the optimization process. The resulting performance curves, in the form of geometrical rms image radius versus field angle, are shown in Fig. 2 for this parametric family of optimal designs. The individual curves in Fig. 2 are designated by the field parameter $\theta_{B}$, the intermediate angle at which the rms image radius was minimized. Note that the locus of minima for this family of curves is a straight line with nonzero slope on this plot of rms image radius versus field angle. We thus interpret this empirical ray trace data as indicating that the shaded area represents an uncorrectable linear coma-like aberration. This is consistent with Nariai's conclusion that coma can be minimized, but not eliminated with a hyperboloid-hyperboloid grazing incidence $\mathrm{x}$-ray telescope design. ${ }^{14,15}$ The nonzero on-axis values of rms image radius clearly represent some combination of defocus and spherical aberration. These two-mirror systems are inherently free of chromatic aberration, and the Petzval field curvature is primarily determined by the first-order properties of the design and therefore does not change significantly among the various designs. We also know that these grazing incidence telescopes suffer from severe astigmatism and oblique spherical aberration. ${ }^{6-9}$ Since the fifth-order oblique spherical aberration has the same field dependence as third-order astigmatism and the same pupil dependence as third-order spherical aberration, ${ }^{16}$ it is reasonable to interpret each of the above designs as having balanced defocus, field curvature, thirdorder spherical aberration, third-order astigmatism, and oblique spherical aberration, leaving only linear coma (and higher-order aberrations) at the unique field angle $\theta_{B}$. We have thus made optimum use of our five independent design variables. Incidentally, only the first member of this family of hyperboloid-hyperboloid designs is near-aplanatic (exhibiting only a very small amount of spherical aberration and coma), and it is the design that would be optimum for a very small operational field of view (OFOV). Each member of the family of optimal designs was shown to be the optimum design for a given operational field of view $(\mathrm{OFOV}){ }^{12}$

\section{Systems Engineering Analysis of Image Quality}

Making a list of all possible error sources affecting the final performance of a precision imaging system, then categorizing those error sources into groups and constructing an error budget tree, is usually the first step in performing a complete systems engineering analysis of image quality. Figure 3 is an example of an error budget tree for the SXI being built by the National Oceanographic and Atmospheric Administration (NOAA) for use on the nextgeneration Geostationary Operational Environmental Satellite (GOES) weather satellites. ${ }^{13}$

SXI is a staring wide-field grazing incidence x-ray telescope that will be pointed at the center of the sun and will produce full solar-disk images at $\mathrm{x}$-ray wavelengths. Like most current state-of-the-art precision optical systems, the SXI utilizes a mosaic detector array in the focal plane to record the images. Frequently, such optical systems are designed to produce the best possible on-axis aerial image in the focal plane, and the detector effects are included only in the analysis of the final system performance. It is the goal of this paper to demonstrate that including detector effects in the optical design (selection) process can yield significantly improved optical system performance for many wide-field imaging applications.

For wide-field imaging systems it is not uncommon for detector effects to be the limiting factor in determining image quality for small field angles; whereas, geometrical aberrations may be the limiting factor in determining the image quality at large field angles. Most conventional optical design codes are based upon geometrical ray trace analysis and do not have a routine capability for quantitatively modeling detailed detector effects. Furthermore, the grazing incidence $\mathrm{x}$-ray telescope designs have an annular aperture with an extremely high obscuration ratio $(\varepsilon=0.98) .{ }^{10}$ At these very large obscuration ratios, conventional optical design codes do not produce meaningful ray intercept plots, nor do they provide meaningful aberration coefficients. However, if one traces sufficiently dense rays (to adequately fill the very narrow annular aperture) they do produce meaningful spot diagrams. We have thus imported our exhaustive ray trace results into a MATLAB code where they have been combined with a detailed modeling of diffraction effects, surface scatter effects, and all of the other miscellaneous residual errors in the mirror manufacturer's error budget tree shown in Fig. 3. This yields a systems engineering analysis of the quality of the aerial image produced in the telescope focal plane. ${ }^{10,17}$ However, as indicated by Fig. 3, we have yet to accurately model the detector effects. And we want to include those detector effects in the optical design selection process (recall that the entire family of candidate optical designs have already been produced).

Our optical design process thus consists of including a detailed analysis of detector effects upon the aerial images produced by the predetermined parametric optimal family of designs, then selecting the particular optical design that is optimum (based upon our image quality criterion) for a given application. 


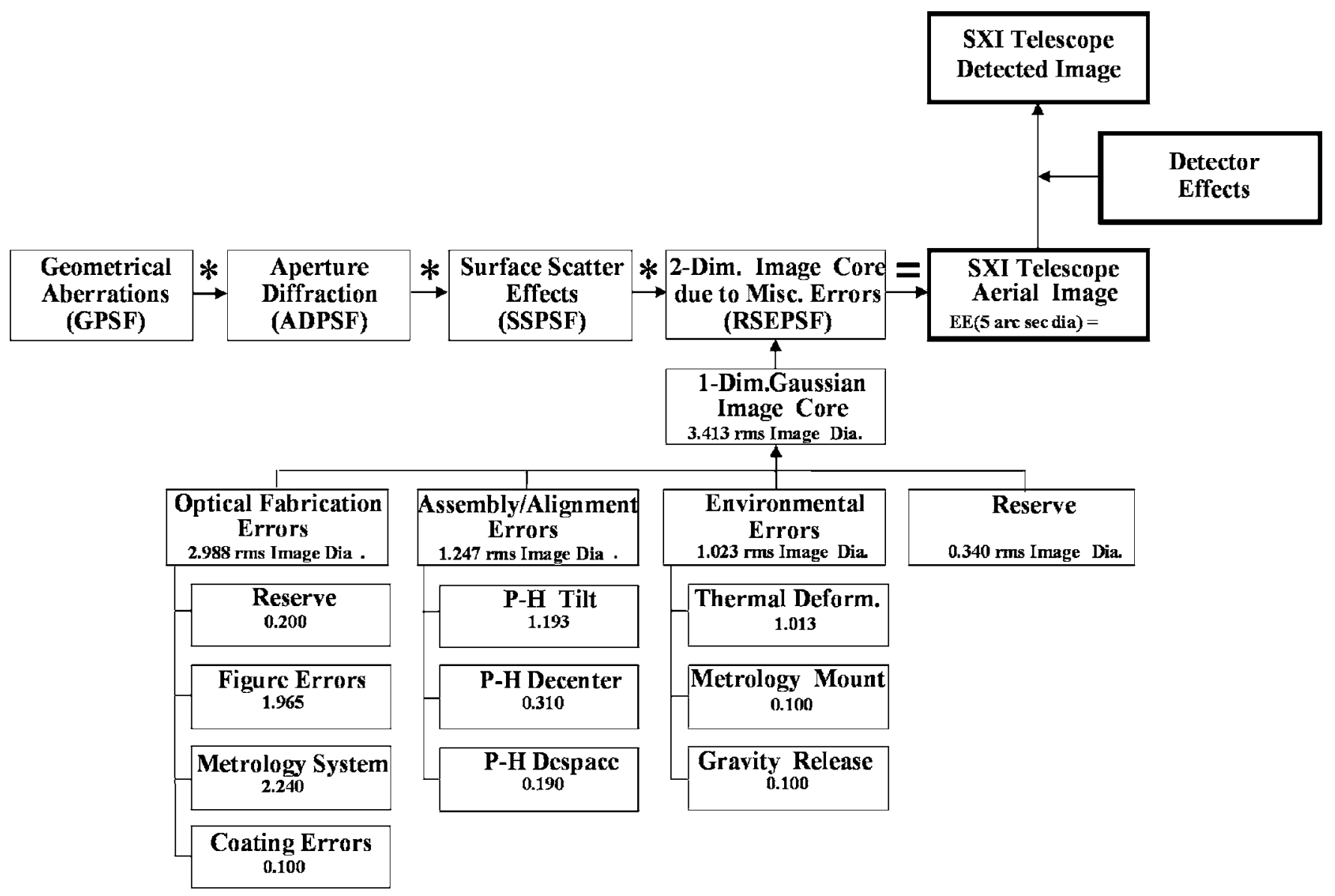

Fig. 3 Error budget tree, indicating the usual practice of considering detector effects only after the imaging system has been designed to produce the best possible aerial image.

\section{Detection with Mosaic Detector Arrays}

The modulation transfer function (MTF) is widely used in the initial specification and design of many imaging systems, as well as in the subsequent detailed analysis of the images they produce. However, implicit in this is the mathematical assumption that the imaging system is both linear and shift-invariant, i.e., that the location (and strength) of a point source can be chosen arbitrarily.

When a single detector is scanned over an aerial image, the detected image (in the scan direction) can be modeled by the convolution of the aerial image with the detector pixel; or conversely, one can multiply the MTF of the imaging system by the detector MTF. However, these linescan devices all employ a discrete sampling interval in the direction perpendicular to the scan direction, and the MTF approach to system performance analysis is not directly applicable to these scanning techniques or imaging systems utilizing staring mosaic detector arrays. The sampling causes these systems to exhibit a particular kind of local shift variance, which causes the appearance of the reconstructed image to vary with the location of the aerial PSF relative to the sampling (i.e., pixel) grid. ${ }^{18,19}$

For example, in an imaging system utilizing a staring mosaic detector array, the aerial image is sampled (averaging over each detector pixel) to produce a detected point spread function (DPSF). An interpolation scheme can then be used to reconstruct a smooth DPSF; however, the detailed characteristics of the DPSP vary substantially with the registration (or lack thereof) of the aerial PSF on a given detector pixel. In other words, the imaging process using a staring mosaic detector array is not a shift-invariant process. This detector registration (or alignment) process must therefore be discussed in some detail.

Assuming a Gaussian aerial PSF slightly larger than a detector pixel, Fig. 4 illustrates the resulting DPSF and reconstructed DPSF for the following three situations: (1) when the aerial PSF is precisely "registered" at the center of a detector pixel, (2) when the aerial PSF is positioned on the boundary between two detector pixels, and (3) when the aerial PSF is positioned at a point where four detector pixels meet.

If the detector array is not "registered" we get substantially different quantitative results for various characteristics of the reconstructed DPSF. For example, the half power radius (HPR) of the reconstructed DPSF can increase by more than $40 \%$ over the registered value. For an application where the telescope is being operated as a staring telescope recording fine detail in an extended image (random location of aerial PSF on pixel), the "average unregistered" detected point spread function (AUDPSF) is given by the convolution of the registered detected point spread function (RDPSF) with the unit cell of the sampling grid. ${ }^{19}$ 


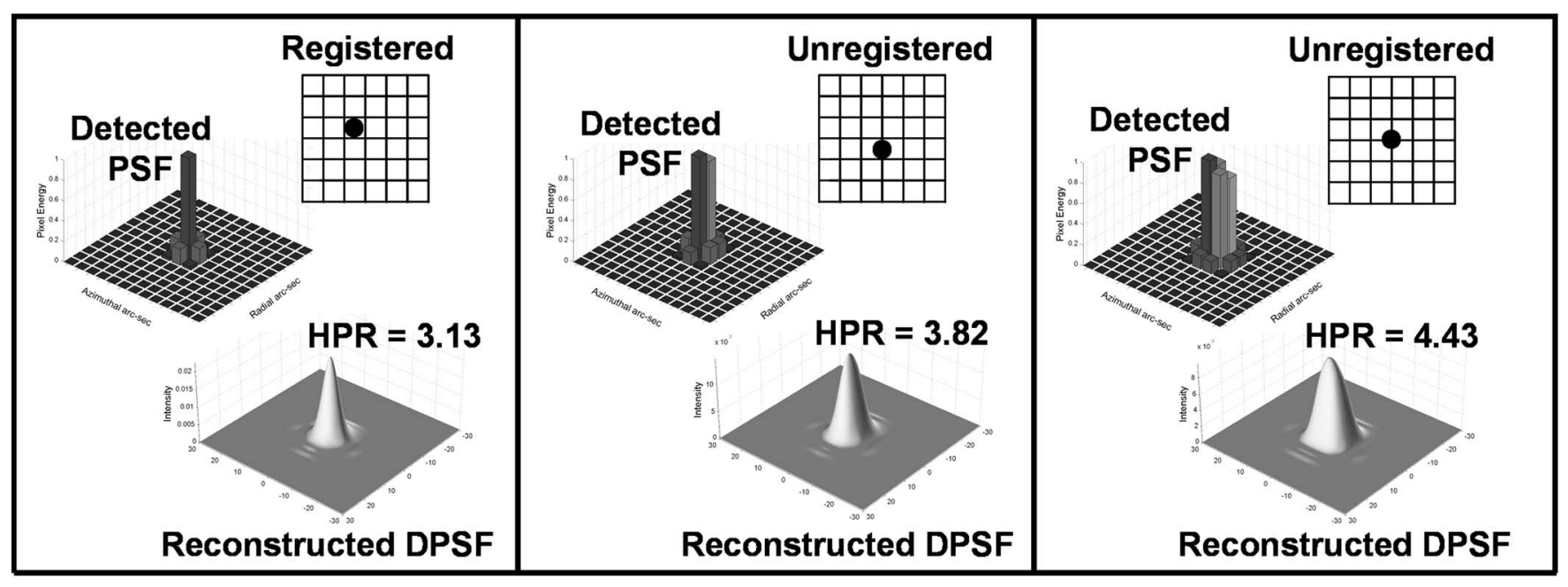

(a)

(b)

(c)

Fig. 4 The detected PSF and the reconstructed DPSF for. (a) precisely "registered" aerial PSF, (b) aerial PSF centered on boundary between two pixels, and (c) aerial PSF positioned where four pixels meet.

The calculation of both the reconstructed registered DPSF and the reconstructed average unregistered DPSF are thus illustrated in Fig. 5. Since the aerial PSF is represented as a dense numerical array, the averaging over the individual pixels is referred to as a "binning" operation. Care is taken to precisely "register" the sampling detector grid by positioning it so as to maximize the signal produced by a given pixel. We then use a cubic interpolation technique to reconstruct the "registered" DPSF. Finally, we convolve with the unit cell of the sampling grid to produce the average unregistered DPSF.

\section{Image Quality Criteria for Wide-field Imaging Systems}

It is particularly important that the appropriate image quality criterion be chosen for a specific application, as it can be quite costly and time-consuming to change the top-level image quality requirement halfway through a major program. For example, the FWHM is an appropriate image quality criterion for a telescope that is going to be used to resolve bright binary stars (i.e., signal-to-noise ratio is not a problem). Fractional encircled energy is a particularly ap-

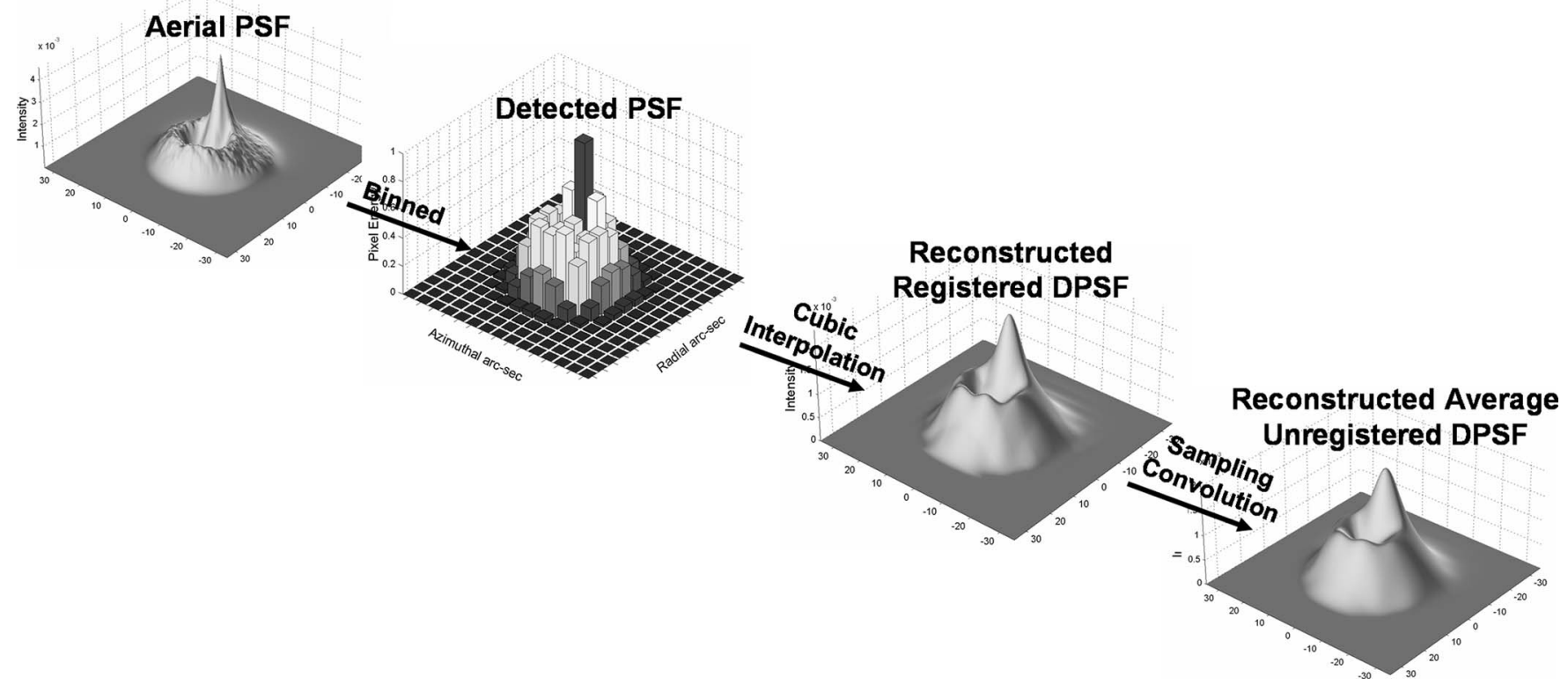

Fig. 5 A graphical illustration of the numerical computation technique for modeling both the reconstructed "registered" DPSF and the reconstructed "average unregistered" DPSF is indicated. 


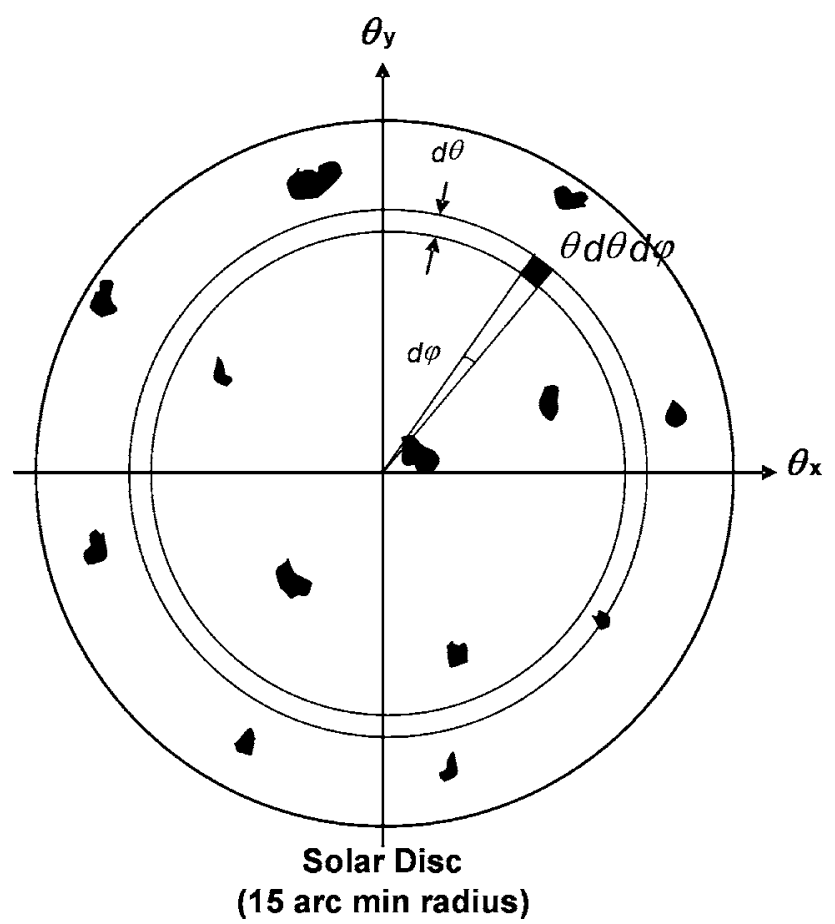

Fig. 6 Graphical illustration of the solar disc with randomly placed sunspots or solar flares.

propriate image quality criterion for a telescope whose main function is collect radiant energy and concentrate it upon the entrance slit of a spectrographic instrument. And some characteristic of the MTF is often more appropriate for an imaging system that will be used to study fine detail in an extended image.

However, the image quality criterion for a wide-field imaging application should be expressed in terms of some field-weighted-average "resolution" over a predetermined OFOV. ${ }^{11-20}$ This is certainly the case for the SXI telescope operating in a staring mode, recording and transmitting full solar disk-images of solar flare activity for study by NOAA scientists and solar physicists. Specifically, we will choose the field-weighted-average half power radius $\left(\mathrm{HPR}_{\mathrm{fwa}}\right)$ of the DPSFs discussed in the previous section.

$\mathrm{HPR}_{\mathrm{fwa}}=\frac{1}{A_{T}} \int_{\theta=0}^{\mathrm{OFOV}} \operatorname{HPR}(\theta) 2 \pi \theta d \theta$,

where $A_{T}=\pi(\mathrm{OFOV})^{2}$.

Figure 6 illustrates the full solar disk, which has an angular radius of approximately 15 arc min. The OFOV will want to be considerably greater than 15 arc min since some solar flares and coronal mass ejections extend somewhat beyond the solar limb. The total number of spatial resolution elements in the OFOV is closely related to the above merit function, and is given by the following equation:

$N=\#$ of Res. Ele. $=2 \int_{\theta=0}^{\mathrm{OFOV}} \frac{\theta}{\operatorname{HPR}^{2}(\theta)} d \theta$.

Since sunspots or solar flares have an equal probability of appearing anywhere on the solar disk, the total information

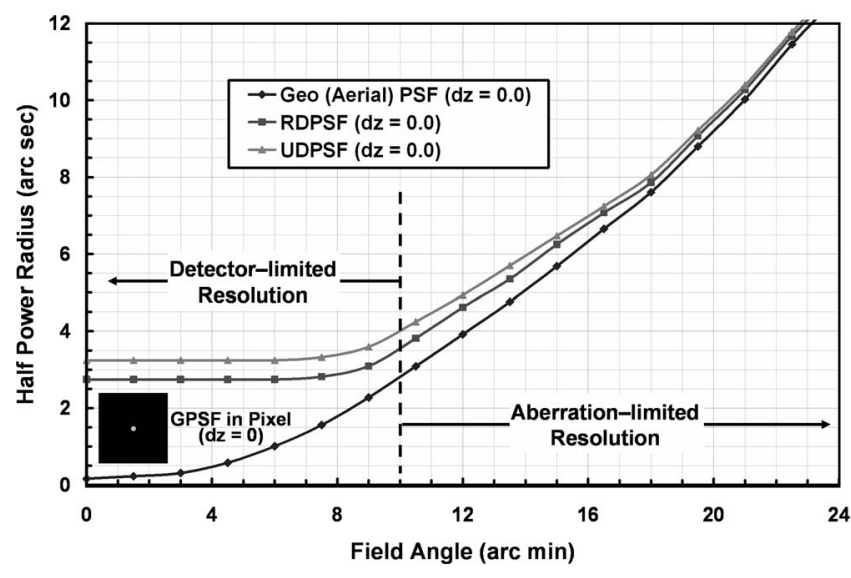

Fig. 7 Illustration of detector effects for a near-aplanatic grazing incidence $x$-ray telescope design.

content of a given snapshot of the solar disk is maximized if we minimize the field-weighted-average resolution element as degraded by all error sources. In particular, if the SXI telescope is not going to be routinely pointed to the particular feature of interest, this image quality criterion is vastly superior to one that maximizes the on-axis image quality.

\section{Including Detector Effects in the Image Analysis}

We have previously developed a family of optimal grazing incidence hyperboloid-hyperboloid x-ray telescope designs, where each member of the family is the optimum design for a different OFOV. ${ }^{15}$ Figure 7 illustrates the HPR of the geometrical PSF versus field angle for a near-aplanatic hyperboloid-hyperboloid grazing incidence $\mathrm{X}$-ray telescope design with the SXI first-order design parameters. Included in the same graph is the HPR versus field angle of the RDPSF and the AUDPSF where we have assumed the $15.8 \mu \mathrm{m}$ (5.0 arc sec) detector pixels to be used in the SXI instrument. Note that the image quality is clearly "detectorlimited" for small field angles and "aberration-limited" for large field angles.

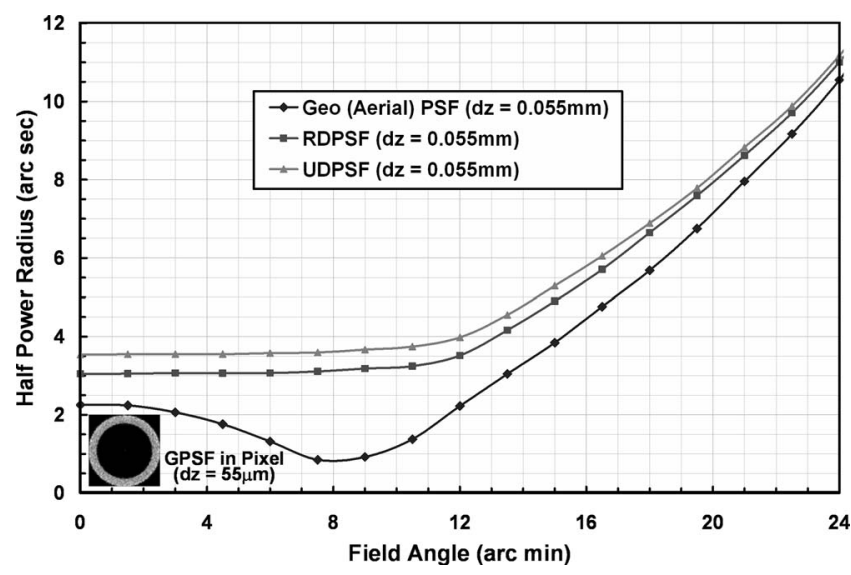

Fig. 8 Illustration of detector effects for a despaced near-aplanatic grazing incidence $x$-ray telescope design. 

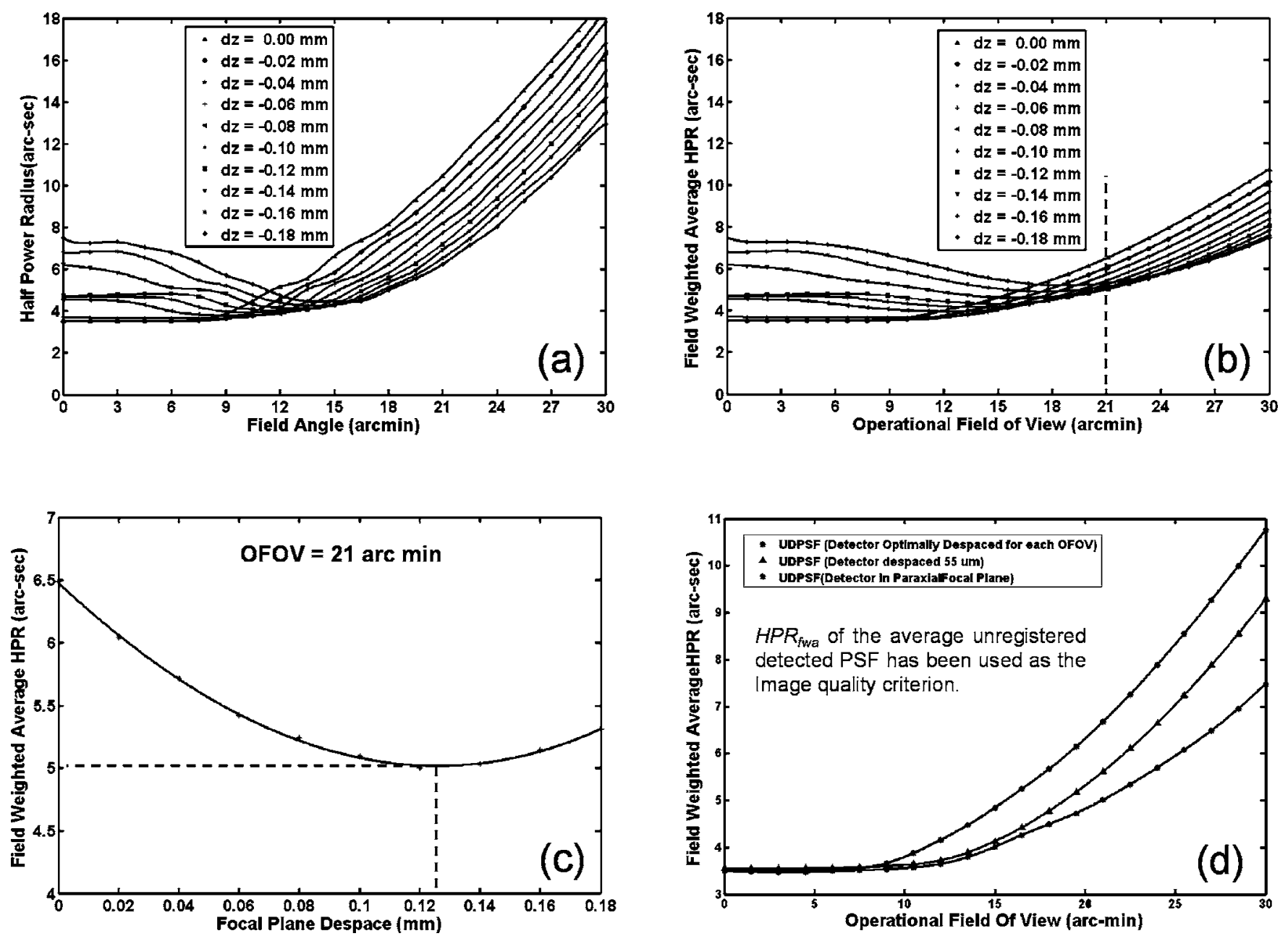

Fig. 9 (a) Illustration of the HPR versus field angle of the AUDPSF for a variety of focal plane despace values, (b) $\mathrm{HPR}_{\mathrm{fwa}}$ versus OFOV for the same data, (c) $\mathrm{HPR}_{\mathrm{fwa}}$ versus focal plane despace for OFOV $=21$ arc sec, and (d) additional improvement in image quality when the focal plane despace is optimized for each OFOV.

It is common practice to despace the operational focal plane of a wide-field imaging system to balance field curvature with defocus, thus improving wide-field image quality (of the aerial image) at the expense of small-field image quality. However, when detector effects are included, there is virtually no small-field image degradation due to this despacing operation until the resulting defocused on-axis geometrical PSF exceeds the detector pixel size. A despace of $55 \mu \mathrm{m}$ is allowed in the SXI design before the defocused geometrical PSF (annulus) completely fills a detector pixel.

Figure 8 illustrates the HPR of the geometrical PSF versus field angle for the despaced near-aplanatic hyperboloidhyperboloid grazing incidence $\mathrm{x}$-ray telescope design, and again compares it with the HPR versus field angle of the RDPSF and the AUDPSF. Note that the geometrical performance (no detector effects) is improved substantially for large field angles at the expense of degraded small-field performance. And indeed, when detector effects are included, the wide-angle performance is improved substantially with virtually no additional degradation at small field angles.
We will now determine precisely what value of despace will minimize the field-weighted-average HPR defined in Eq. (1). Using the near-aplanatic grazing incidence $\mathrm{x}$-ray telescope design defined by the Zemax lens editor values listed in Table 1, we performed extensive ray trace analyses and calculated the HPR of the AUDPSF for a variety of field angles and despace values as illustrated in Fig. 9(a). We then used Eq. (1) and performed a two-dimensional integration of this data to produce the $\mathrm{HPR}_{\mathrm{fwa}}$ versus OFOV curves illustrated in Fig. 9(b). Note that the on-axis ordinate values of these two sets of curves are the same, but the off-axis values of the curves in Fig. 9(b) are substantially reduced due to the averaging process.

Finally, to find the optimum despace for a given OFOV, we plotted the $\mathrm{HPR}_{\mathrm{fwa}}$ at that OFOV as a function of despace. This curve is shown in Fig. 9(c) for an OFOV of 21 arc min. From the curve in Fig. 9(c) it is obvious that the optimum focal plane despace for a 21 arc min OFOV is about $124 \mu \mathrm{m}$. This value of despace produces an $\mathrm{HPR}_{\mathrm{fwa}}$ of about 5.0 arc sec, down from 6.5 arc sec for the paraxial focal plane. This procedure has been repeated for different OFOVs to obtain a plot of the optimally despaced $\mathrm{HPR}_{\mathrm{fwa}}$ 


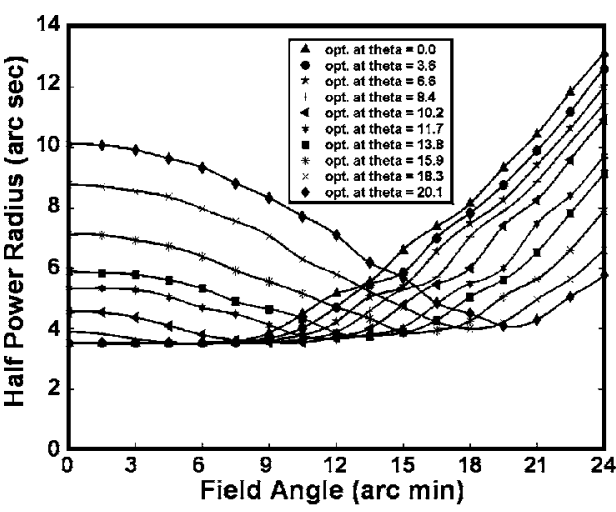

(a)

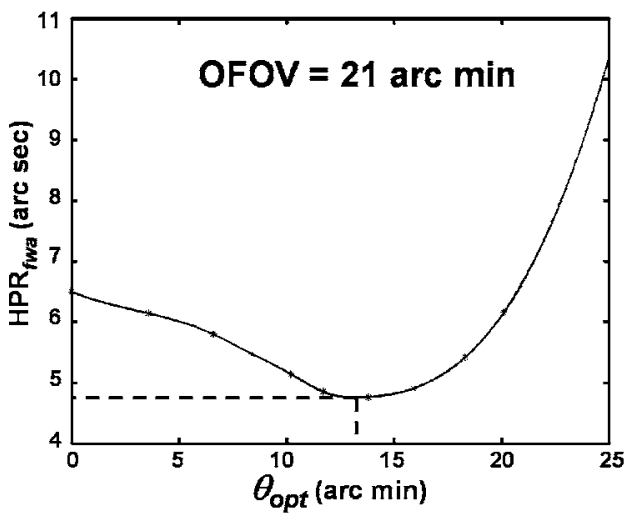

(c)

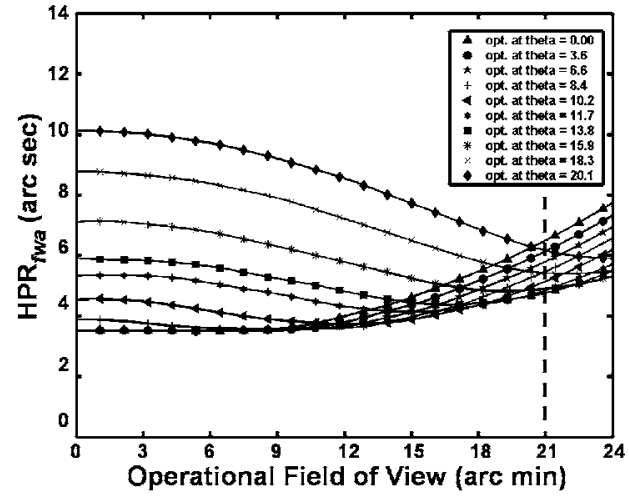

(b)

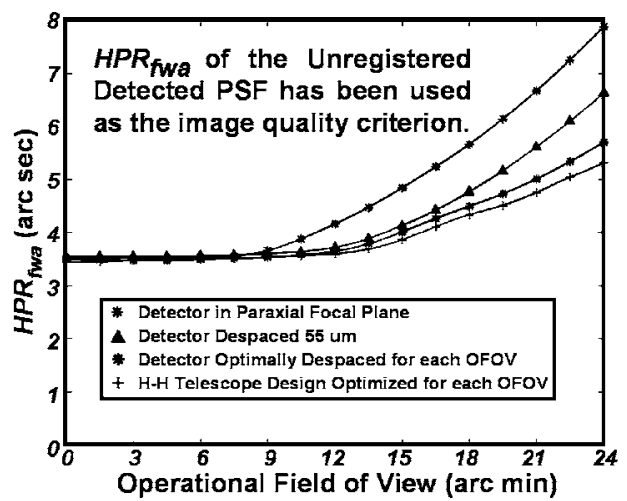

(d)

Fig. 10 (a) Illustration of the HPR versus field angle of the AUDPSF for a variety of optimal optical designs, (b) $\mathrm{HPR}_{\mathrm{fwa}}$ versus OFOV for the same data, (c) $\mathrm{HPR}_{\mathrm{fwa}}$ versus optical design parameter $\theta_{B}$ for $O F O V=21$ arc sec, and (d) illustration of additional improvement in image quality when the optical design is optimized for each OFOV.

as a function of the OFOV. In Fig. 9(d), this curve is compared to the performance of the system when the detector is despaced by $55 \mu \mathrm{m}$ and when the mosaic detector array is positioned in the paraxial focal plane.

\section{Including Detector Effects in the Optical Design Process}

In Ref. 12 we developed a whole family of hyperboloidhyperboloid grazing incidence $\mathrm{x}$-ray telescope designs where each member of the family provided optimum geometrical performance for a different OFOV. The resulting geometrical performance curves were illustrated in Fig. 2. The design designated as $\theta_{B}=12.1$ yields geometrical image sizes that are nearly the same on-axis and at a field angle of 16.5 arc min, slightly above the solar limb, and is thus similar to the design chosen for the SXI mission. The design chosen for the SXI mission optimized the aerial image over an OFOV with a radius of 18 arc min.

We will now apply a procedure similar to that used in the previous section and include detector effects in the optical design selection process. This will yield yet additional improvement in the image quality over that obtained by optimally despacing the focal plane of an aplanatic optical design.

Figure 10(a) illustrates the HPR of the AUDPSF for a variety of field angles and despace values. We again used Eq. (1) and performed a two-dimensional integration of this data to produce the $\mathrm{HPR}_{\mathrm{fwa}}$ versus $\mathrm{OFOV}$ curves illustrated in Fig. 10(b). To find the optimum optical design for a given OFOV, we again plotted the $\mathrm{HPR}_{\mathrm{fwa}}$ at that OFOV as a function of the parameter $\theta_{B}$ which defines the different members of the family of optimal optical designs. This curve is shown in Fig. 10(c) for an OFOV of 21 arc min. From the curve in Fig. 10(c) we see that the optimum optical design for a 21 arc min OFOV is designated by $\theta_{B}$ $=14 \mathrm{arc} \mathrm{min}$. This procedure has been repeated for different OFOVs to obtain a plot of the optimal hyperboloidhyperboloid grazing incidence $\mathrm{x}$-ray telescope designs as a function of the OFOV. This curve is illustrated in Fig. 10(d) and compared to the performance of the system when an aplanatic optical design is used with the detector optimally despaced for each OFOV, when an aplanatic optical design despaced by $55 \mu \mathrm{m}$, and when the mosaic detector array is positioned in the paraxial focal plane of an aplanatic optical design.

Minimizing the $\mathrm{HPR}_{\mathrm{fwa}}$ over a given OFOV will maximize the number of resolution elements $N$ over that OFOV. And, of course, increasing the number of angular resolution elements over the OFOV increases the amount of information in the image. Figure 11 illustrates the number of angular resolution elements as a function of the OFOV for the four situations considered in this paper: (a) a near-aplanatic grazing incidence $\mathrm{x}$-ray telescope design with the SXI firstorder design parameters having a mosaic detector array in 


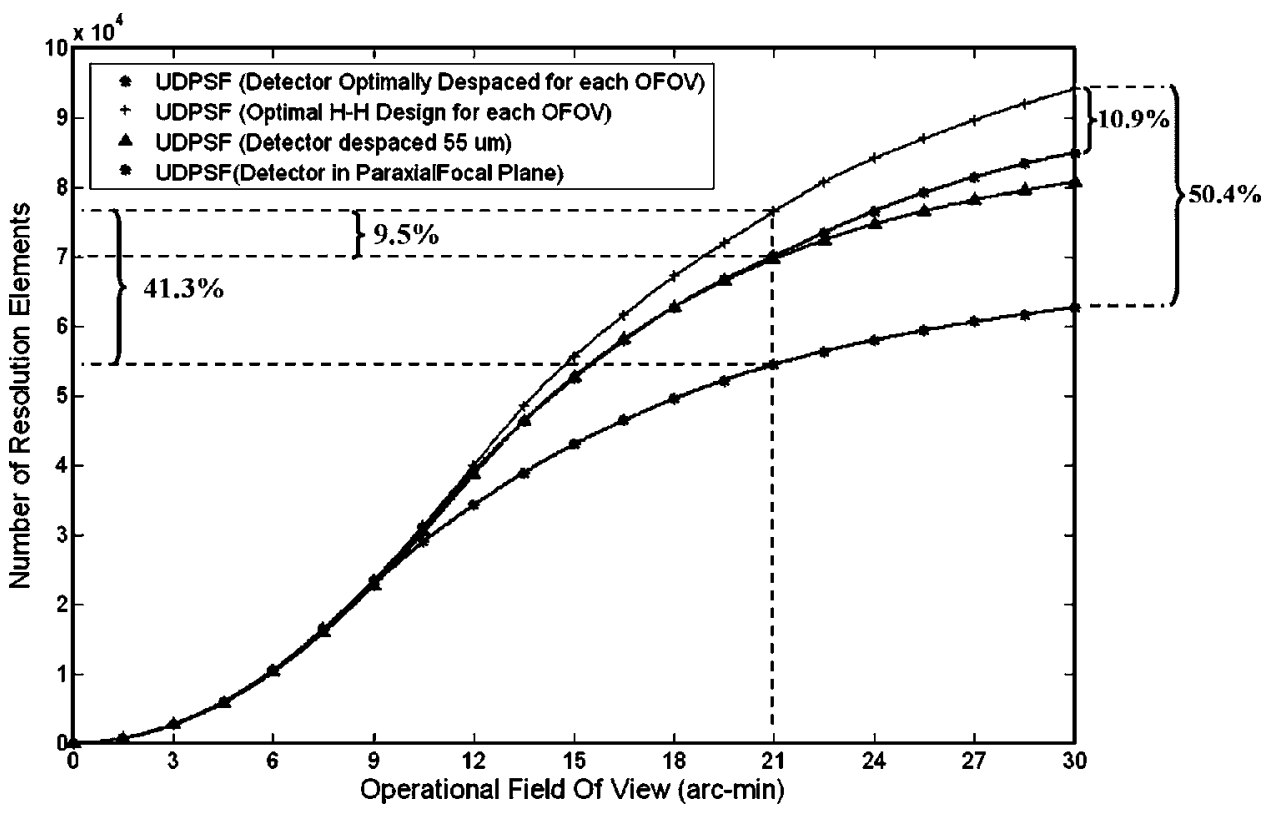

Fig. 11 Illustration of the number of resolution elements $N$ versus OFOV for the AUDPSF for four different situations, showing the improvement in image quality when the optical design is optimized for each OFOV.

the paraxial focal plane, (b) the same near-aplanatic grazing incidence $\mathrm{x}$-ray telescope design with the mosaic detector array despaced until the defocused geometrical PSF just fills a detector pixel, (c) the same optical design with the mosaic detector array optimally despaced for each OFOV, and finally (d) having the optimal nonaplantic hyperboloidhyperboloid optical design for each OFOV.

From the curves in Fig. 11 we conclude that: (a) for OFOV $<9$ arc min, the detector effects are so dominant that all four situations provide the same result; i.e., there is no penalty in performance for using the classical Wolter type I design (no advantage to the aplanatic design); (b) for $9<$ OFOV $<21$ arc min, despacing the detector until the geometrical PSF just fills a detector significantly improves the system performance; (c) for OFOV $>21$ arc min, optimally despacing (by more than $55 \mathrm{~mm}$ ) the detector for each OFOV yields even more improvement in wide-field performance with no loss in small-field performance. However, for OFOV $>12$ arc min, further substantial improvement in optical performance can be obtained by balancing detector effects with geometrical aberrations. This requires a different optimum (nonaplanatic) hyperboloidhyperboloid optical design for each OFOV. More specifically, we see that for a 30 arc min OFOV, the optimum optical design yields a $50.4 \%$ increase in the number of angular resolution elements over that obtained with an aplanatic design with the mosaic detector array located in the paraxial focal plane, and a $10.9 \%$ improvement over the

Table 1 ZEMAX lens editor values for SXI baseline design.

\begin{tabular}{rcccccc}
\hline \hline Surface & Type & Radius & Thickness & Glass & Semidiameter & Conic \\
\hline OBJ & Standard & Infinity & Infinity & - & Infinity & 0 \\
$1^{*}$ & Standard & Infinity & 75 & - & 81.9494848999 & 0 \\
2 & Standard & Infinity & 50 & - & 100 & 0 \\
3 & Standard & Infinity & 50 & - & 100 & 0 \\
4 & Standard & Infinity & 1320.19681220 & - & 100 & 0 \\
STO* & Standard & -2.2653939800 & -722.52414458 & Mirror & 81.4913300000 & -1.0001024853 \\
$6 *$ & Alternate-even & -2.5964833000 & 7.4272985506 & Mirror & 80 & -1.0072425757 \\
7 & Standard & Infinity & 0 & - & 10 & 0 \\
IMA & Alternate-even & Infinity & - & - & 10 & 0 \\
\hline \hline
\end{tabular}


aplanatic design with an optimally despaced detector array. The improvement clearly decreases with decreasing OFOV. For example, for a 21 arc min OFOV there is approximately a $41.3 \%$ increase in performance over the aplanatic design with the detector in the paraxial focal plane and a 9.5\% increase over the aplanatic design with an optimally despaced detector array. These quantitative predictions are the result of a refinement in the calculations presented in an earlier SPIE conference proceedings. ${ }^{21}$

\section{Summary and Conclusions}

We have analyzed the effects of a mosaic detector array upon the optical performance of a grazing incidence $\mathrm{x}$-ray telescope to be utilized in a wide-field imaging application. After choosing an appropriate image quality criterion for wide-field imaging applications, we demonstrated a procedure for determining the optimum focal plane position for an aplanatic telescope design covering an arbitrary OFOV. We then extended the procedure to include detector effects in the optical design process and showed that a significant improvement in optical performance can be achieved over an aplanatic design with an optimally despaced focal plane. These results clearly demonstrate that there is little merit in an aplanatic optical design for some applications utilizing a mosaic detector array (with practical pixel sizes) in the focal plane. This is a direct result of the fact that detector effects dominate all geometrical aberrations at small field angles; whereas field curvature, astigmatism, and higherorder off-axis aberrations dominate spherical aberration and coma at large field angles. Thus correcting spherical aberration and coma merely use up precious optical design variables without exhibiting any improvement in system performance.

\section{Acknowledgments}

This work was supported in part by the Lockheed Martin Solar and Astrophysical Laboratory (LMSAL) under the NOAA Solar X-ray Imager (SXI) program.

\section{References}

1. N. M. Ceglio, "Revolution in x-ray optics," J. X-Ray Sci. Technol. 1, 7-78 (1989).

2. H. Wolter, "Mirror systems with glancing incidence on image producing optics for x-rays," Ann. Phys. 10, 94-114 (1952).

3. L. VanSpeyroeck and R. C. Chase, "Design parameters of paraboloidhyperboloid telescopes for x-ray astronomy," Appl. Opt. 11(2), 440445 (1972).

4. H. Wolter, "Generalized Schwarzschild mirror systems with glancing incidence as optics for x-rays," Ann. Phys. 10, 286-295 (1952).

5. R. C. Chase and L. VanSpeyroeck, "Wolter-Schwarzschild telescopes for x-ray astronomy," Appl. Opt. 12(5), 1042-1044 (1973).

6. W. Werner, "Imaging properties of wolter I type x-ray telescopes," Appl. Opt. 16, 764-773 (1977).

7. T. T. Saha, "Transfer ray aberrations of Wolter type I telescopes," Proc. SPIE 640, 10-19 (1986).

8. T. T. Saha, "Aberrations for grazing incidence telescopes," Appl. Opt. 27, 1492-1498 (1988).

9. D. L. Shealy and T. T. Saha, "Formula for the rms blur circle radius of Wolter telescope based on aberration theory," Appl. Opt. 29, 24332439 (1990).

10. P. L. Thompson and J. E. Harvey, "Systems engineering analysis of aplanatic Wolter type I x-ray telescopes," Opt. Eng. 39, 1677-1691 (June 2000).

11. P. L. Thompson and J. E. Harvey, "Development of an image perfor- mance criterion for wide-field grazing incidence x-ray telescopes," Proc. SPIE 3766, 162-172 (1999).

12. J. E. Harvey, A. Krywonos, P. L. Thompson, and T. T. Saha, "Grazing incidence hyperboloid-hyperboloid designs for wide-field x-ray imaging applications," Appl. Opt. 40, 136-144 (January 2001).

13. P. L. Bornman, D. Speich, J. Hirman, V. Pizzo, R. Grubb, C. Balch, and G. Heckman, "GOES Solar X-ray Imager: overview and operational goals," Proc. SPIE 2812, 309-319 (1996).

14. K. Nariai, "Geometrical aberrations of a generalized Wolter type I telescope," Appl. Opt. 26, 4428-4432 (1987).

15. K. Nariai, "Geometrical aberrations of a generalized Wolter type I telescope. 2: Analytical study," Appl. Opt. 27, 345-350 (1988).

16. V. N. Mahajan, Optical Imaging and Aberrations Part I: Ray Geometrical Optics, SPIE Optical Engineering Press, Bellingham, WA (1998).

17. J. E. Harvey, M. Atanassova, and A. Krywonos, "Systems engineering analysis of five "as-manufactured" SXI telescopes," Proc. SPIE 5867, 5867F (Aug. 2005)

18. S. K. Park, R. Schowengerdt, and M.-A. Kaczynski, "Modulationtransfer-function analysis for sampled image systems," Appl. Opt. 23, 2572-2582 (Aug. 1984).

19. G. D. Boreman, Modulation Transfer Function in Optical and Electro-Optical Systems, Tutorial Texts in Optical Engineering Vol. TT52, pg. 41, SPIE Press, Bellingham, WA (2001).

20. C. J. Burrows, R. Burg, and R. Giacconi, "Optimal grazing incidence optics and its application to wide-field x-ray imaging," Astrophys. $J$. 392, 760-765 (1992).

21. J. E. Harvey, M. Atanassova, and A. Krywonos, "Including detector effects in the design of wide-angle imaging systems," Proc. SPIE 5523, 90-99 (Aug. 2004).

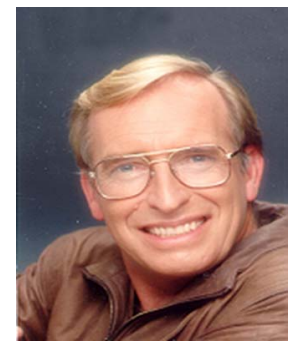

James E. Harvey received his $\mathrm{PhD}$ from the University of Arizona in 1976. He is currently an associate professor in the College of Optics and Photonics at the University of Central Florida and a senior staff member at the Center for Research and Education in Optics and Lasers (CREOL). Dr. Harvey is credited with more than one hundred publications and conference presentations in the areas of diffraction theory, surface scatter phenomena, adaptive optics, wavefront sensing, beam sampling technology, optical properties of materials, and X-ray EUV imaging systems. He is a member of the OSA and a fellow of SPIE.

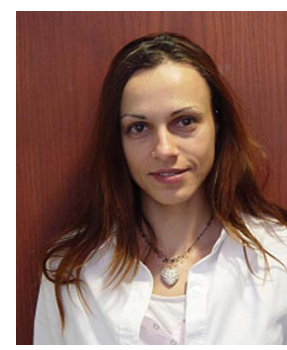

Martina Ivanova Atanassova received her BS degree in engineering physics from Sofia University in Bulgaria in 1997, and an MS degree in engineering physics from Sofia University in Bulgaria in 1999. She has just completed her PhD in Optics from the College of Optical and Photonics at the University of Central Florida. This paper results from the research leading to her PhD dissertation, which was performed in the Optical Design and Image Analysis Laboratory of the Center for Research and Education in Optics and Lasers (CREOL).

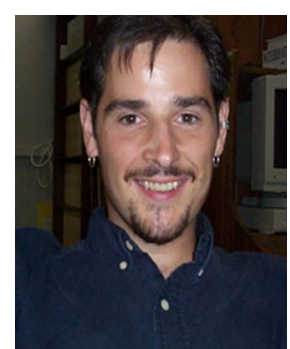

Andrey Krywonos received a bachelor's degree in photonics from the State University of New York Institute of Technology at Utica, New York, in 1995 and a master's degree in optics from the University of Central Florida in 2000 . He is currently a PhD candidate in the College of Optics and Photonics at the University of Central Florida working in the area of surface scatter and image analysis. 